DOI: $10.15393 / \mathrm{j} 3 . \operatorname{art} .2019 .6730$

UDC 517.956.224

V. N. DUBinin

\title{
GREEN ENERGY AND EXTREMAL DECOMPOSITIONS
}

\begin{abstract}
We give two precise estimates for the Green energy of a discrete charge, concentrated in an even number of points on the circle, with respect to the concentric ring. The lower estimate for the Green energy is attained for the points with a nonstandard symmetry. The well-known Pólya-Schur inequality for the logarithmic energy is a special case of this estimate. The proof is based on the application of dissymmetrization and an asymptotic formula for the conformal capacity of a generalized condenser in the case when some of its plates contract to given points. The upper bound is established for a charge that takes values of opposite signs. Its proof reduces to solving a problem on the so-called extremal decomposition of a circular ring with free poles on a circle.
\end{abstract}

Key words: Green energy, discrete charge, dissymmetrization, extremal decompositions

\section{Mathematical Subject Classification: $31 A 15$}

1. Introduction and statement of results. There are many studies related to the extremal problems for different kinds of energy of a discrete charge (see, e.g., the papers [2-4], [9], [10], [12], and the references therein). In contrast to the previous research, we consider the Green energy (see also the recent articles [1] and [5]). In addition, an extremal problem with an alternating charge is studied.

Let $\theta_{k}, k=1, \ldots, 2 n$, be real numbers, such that

$$
\theta_{1}<\theta_{2}<\ldots<\theta_{2 n}<\theta_{1}+2 \pi
$$

$(n \geqslant 2)$, and let $Z=\left\{z_{k}\right\}_{k=1}^{2 n}, z_{k}=\exp \left(i \theta_{k}\right), k=1, \ldots, 2 n$. Denote by

$$
E(Z, B)=\sum_{k=1}^{2 n} \sum_{\substack{l=1 \\ l \neq k}}^{2 n} g_{B}\left(z_{k}, z_{l}\right)
$$

(C) Petrozavodsk State University, 2019 
the Green energy of the collection $Z$ with respect to the ring $B:=\left\{z: \quad R_{1}<|z|<R_{2}\right\}, 0<R_{1}<1<R_{2}<\infty$. Here $g_{B}\left(z, z_{k}\right)$ is the Green function of the domain $B$ with poles at $z_{k}, k=1, \ldots, 2 n$.

The following statement is valid.

Theorem 1. For any collection of points $Z$, and for any numbers $R_{1}, R_{2}$, the inequality

$$
E(Z, B) \geqslant E\left(Z^{*}, B\right)
$$

holds, where $Z^{*}=\left\{z_{k}^{*}\right\}_{k=1}^{2 n}$, and the symmetrically located points $z_{k}^{*}$ are defined by the relations $\left|z_{k}^{*}\right|=1, \arg z_{2 j-1}^{*}=-\eta /(2 n)+2 \pi j / n$, $\arg z_{2 j}^{*}=\eta /(2 n)+2 \pi j / n, j=1, \ldots, n, \eta=\sum_{j=1}^{n}\left(\theta_{2 j}-\theta_{2 j-1}\right)$.

The proof of Theorem 1 is based on the theory of the condenser capacities and dissymmetrization. These ideas go back to the book [4]. By taking the limit $\eta \rightarrow 0$ in (1), we obtain an inequality for the Green energy of a charge concentrated in an unrestricted number of points (not necessary even). Passing to the limit $R_{1} \rightarrow 0, R_{2} \rightarrow \infty$, inequality (1) gives the classical Pólya-Schur inequality

$$
\prod_{k=1}^{n} \prod_{\substack{l=1 \\ l \neq k}}^{n}\left|z_{k}-z_{l}\right| \leqslant \prod_{k=1}^{n} \prod_{\substack{l=1 \\ l \neq k}}^{n}\left|z_{k}^{*}-z_{l}^{*}\right|=n^{n}
$$

where $z_{k}, k=1, \ldots, n$, are some points on the unit circle $|z|=1$ and $z_{k}^{*}=\exp (2 \pi i k / n), k=1, \ldots, n$.

Theorem 2. Let $Z$ and $B$ be as above. Then, for the discrete Green energy

$$
\mathcal{E}(Z, B):=\sum_{k=1}^{2 n} \sum_{\substack{l=1 \\ l \neq k}}^{2 n}(-1)^{k+l} g_{B}\left(z_{k}, z_{l}\right)
$$

we have

$$
\mathcal{E}(Z, B) \leqslant \mathcal{E}\left(Z^{*}, B\right)
$$

where $Z^{*}=\left\{z_{k}^{*}\right\}_{k=1}^{2 n}, z_{k}^{*}=\exp (\pi i k / n), k=1, \ldots, 2 n$.

The proof of inequality (2) is carried out by reduction to the extremal decomposition problems, which have a long history and many applications [6], [11]. A significant contribution to the solution of such problems was 
made by N. A. Lebedev [13]. By taking the limit $R_{1} \rightarrow 0, R_{2} \rightarrow \infty$ in (2), we have

$$
\prod_{k=1}^{n} \prod_{\substack{l=1 \\ l \neq k}}^{n}\left|z_{k}-z_{l}\right|^{(-1)^{k+l}} \geqslant\left(\frac{n}{2}\right)^{2 n} .
$$

This inequality has been proved in [7] (see also [6, p. 127]).

2. Proof of Theorem 1. The notions and notation from the book [6] will be used in the proof below. For sufficiently small $r>0$ the condenser

$$
C^{*}(r):=\left(B,\left\{E_{k}^{*}\right\}_{k=0}^{2 n}, \Delta^{*}\right),
$$

where $E_{0}^{*}=\partial B, E_{k}^{*}=\left\{z:\left|z-z_{k}^{*}\right| \leqslant r\right\}, k=1, \ldots, 2 n$, and $\Delta^{*}=$ $=\{0,1, \ldots, 1\}$ is well defined $[6$, p. 33]. Let $\Phi$ be the group of symmetries of $\overline{\mathbb{C}}$, formed by the compositions of the reflections with respect to the rays $\{z: \arg z=2 \pi k / n\}, k=1, \ldots, n$ and the bisectors of the angles formed by these rays ("dihedral group"). The condenser $C^{*}(r)$ is symmetric with respect to the group $\Phi$ ( $\Phi$-symmetric). By Lemma 4.3 from [6], there exist a dissymmetrization Dis, such that Dis $E^{*}=E$. Here

$$
\begin{gathered}
E^{*}=\bigcup_{j=1}^{n}\left\{z:|z|=1,-\frac{\eta}{2 n}+\frac{2 \pi j}{n} \leqslant \arg z \leqslant \frac{\eta}{2 n}+\frac{2 \pi j}{n}\right\}, \\
E=\bigcup_{j=1}^{n}\left\{z:|z|=1, \theta_{2 j-1} \leqslant \arg z \leqslant \theta_{2 j}\right\} .
\end{gathered}
$$

According to Theorem 4.14 [6], we obtain the inequality for the capacities:

$$
\operatorname{cap} C^{*}(r) \geqslant \operatorname{capDis} C^{*}(r) \text {. }
$$

It is clear that

$$
\operatorname{Dis} C^{*}(r)=\left(B,\left\{E_{k}\right\}_{k=0}^{2 n}, \Delta^{*}\right),
$$

where $E_{0}=\partial B$ and $E_{k}=\left\{z:\left|z-z_{k}\right| \leqslant r\right\}, k=1, \ldots, 2 n$.

In view of Theorem 2.1 from [6], the following asymptotic equalities hold as $r \rightarrow 0$ :

$$
\begin{aligned}
\operatorname{cap} C^{*}(r) & =-\frac{4 \pi n}{\log r}- \\
& -2 \pi\left\{\sum_{k=1}^{2 n} \log r\left(B, z_{k}^{*}\right)+E\left(Z^{*}, B\right)\right\}\left(\frac{1}{\log r}\right)^{2}+o\left(\left(\frac{1}{\log r}\right)^{2}\right),
\end{aligned}
$$




$$
\begin{aligned}
& \operatorname{capDis} C^{*}(r)=-\frac{4 \pi n}{\log r}- \\
&-2 \pi\left\{\sum_{k=1}^{2 n} \log r\left(B, z_{k}\right)+E(Z, B)\right\}\left(\frac{1}{\log r}\right)^{2}+o\left(\left(\frac{1}{\log r}\right)^{2}\right),
\end{aligned}
$$

where $r\left(B, z_{k}\right)$ is the inner radius of $B$ with respect to the point $z_{k}$, $k=1, \ldots, n$. Note that

$$
r\left(B, z_{k}^{*}\right)=r\left(B, z_{k}\right), k=1, \ldots, 2 n .
$$

Substituting these equalities in (3), we obtain the required inequality (1).

3. Proof of Theorem 2. Consider at the condenser

$$
\mathcal{C}(r)=\left(B,\left\{E_{k}\right\}_{k=0}^{2 n}, \Delta\right),
$$

where $B, E_{k}, k=0,1, \ldots, 2 n$, as in Section $2, \Delta=\{0,1,-1,1, \ldots,-1\}$. Let $u$ be the potential function of $\mathcal{C}(r)$ [6, p. 13]. In view of the asymptotic formula (2.10) [6], for $r \rightarrow 0$

$$
\begin{gathered}
\int_{B \backslash \cup_{k=1}^{2 n} E_{k}}|\nabla u|^{2}=\operatorname{cap} \mathcal{C}(r)=-\frac{4 \pi n}{\log r}- \\
-2 \pi\left\{\sum_{k=1}^{2 n} \log r\left(B, z_{k}\right)+\mathcal{E}(Z, B)\right\}\left(\frac{1}{\log r}\right)^{2}+o\left(\left(\frac{1}{\log r}\right)^{2}\right) .
\end{gathered}
$$

Consider the family of functions $\left\{u_{k}\right\}_{k=1}^{2 n}$ that are defined in the sectors $\bar{D}_{k}, D_{k}=\left\{z \in B: \theta_{k}<\arg z<\theta_{k+1}\right\}, k=1, \ldots, 2 n$, respectively $\left(\theta_{2 n+1}=\theta_{1}+2 \pi\right)$. For every $k$, the function $u_{k}$ is continuous in $\bar{D}_{k}$, harmonic in $D_{k} \backslash\left(E_{k} \cup E_{k+1}\right)$,

$$
u_{k}(z)=\left\{\begin{array}{l}
(-1)^{k+1}, \quad z \in E_{k} \cap \bar{D}_{k} \\
(-1)^{k+2}, \quad z \in E_{k+1} \cap \bar{D}_{k} ; \\
0, \quad z \in\left(\partial D_{k}\right) \cap(\partial B)
\end{array}\right.
$$

and satisfies conditions: $\partial u_{k} / \partial n=0$ on $\left(\partial D_{k}\right) \cap B \backslash\left(E_{k} \cup E_{k+1}\right)$, $k=1, \ldots, 2 n$. By Dirichlet's principle,

$$
\int_{D_{k} \backslash\left(E_{k} \cup E_{k+1}\right)}|\nabla u|^{2} \geqslant \int_{D_{k} \backslash\left(E_{k} \cup E_{k+1}\right)}\left|\nabla u_{k}\right|^{2}, \quad k=1, \ldots, 2 n .
$$


The symmetry principle for the harmonic functions gives

$$
\int_{D_{k} \backslash\left(E_{k} \cup E_{k+1}\right)}\left|\nabla u_{k}\right|^{2}=\int_{D_{k} \backslash H_{k}}\left|\nabla \omega_{k}\right|^{2},
$$

where $H_{k}=\left\{z:\left|z-\zeta_{k}\right| \leqslant r\right\}, \zeta_{k}=\exp \left(i\left(\theta_{k+1}-\theta_{k}\right) / 2\right)$, and the function $\omega_{k}$ is continuous in $\bar{D}_{k}$, harmonic in $D_{k} \backslash H_{k}$, is equal to 1 on $H_{k}$ and to zero on $\partial D_{k}, k=1, \ldots, 2 n$. Once again, using formula (2.10) [6], we conclude that

$$
\begin{gathered}
\int_{D_{k} \backslash\left(E_{k} \cup E_{k+1}\right)}|\nabla u|^{2} \geqslant \int_{D_{k} \backslash H_{k}}\left|\nabla \omega_{k}\right|^{2}= \\
=-\frac{2 \pi}{\log r}-2 \pi\left[\log r\left(D_{k}, \zeta_{k}\right)\right]\left(\frac{1}{\log r}\right)^{2}+o\left(\left(\frac{1}{\log r}\right)^{2}\right), r \rightarrow 0,
\end{gathered}
$$

$k=1, \ldots, 2 n$. Note that a suitable branch of the $\operatorname{logarithm} w=\log \left(z / \zeta_{k}\right)$ maps the sector $D_{k}$ conformally and univalently onto the rectangle $G_{k}=\left\{w: \log R_{1}<\operatorname{Re} w<\log R_{2},|\operatorname{Im} w|<\left(\theta_{k+1}-\theta_{k}\right) / 2\right\}, k=1, \ldots, 2 n$. The result of the Marcus radial averaging transformation [14] of the family $\left\{G_{k}\right\}_{k=1}^{2 n}$ (with weights $\alpha_{k}=1 /(2 n), k=1, \ldots, 2 n$ ) belongs to the rectangle $G=\left\{w: \log R_{1}<\operatorname{Re} w<\log R_{2},|\operatorname{Im} w|<\pi /(2 n)\right.$ (also, see [6, p. 83] and [8]). By the Marcus theorem,

$$
\prod_{k=1}^{2 n} r\left(D_{k}, \zeta_{k}\right)=\prod_{k=1}^{2 n} r\left(G_{k}, 0\right) \leqslant r^{2 n}(G, 0) .
$$

Taking into account (4) and (5), we find

$$
\sum_{k=1}^{2 n} \log r\left(B, z_{k}\right)+\mathcal{E}(Z, B) \leqslant 2 n \log r(G, 0) .
$$

It is straightforward to see that in the case $z_{k}=\exp (\pi i k / n), k=1, \ldots, 2 n$, we have the equality sign in the last relation. This yields the required inequality.

\section{References}

[1] Beltran C., Corral N., Criado del Rey J. G. Discrete and continuous Green energy on compact manifolds. Journal of Approximation Theory, 2019, vol. 237, pp. $160-185$.

DOI: https://doi.org/10.1016/j.jat.2018.09.004 
[2] Brauchart J. S. Optimal logarithmic energy points on the unit sphere. Math. Comp., 2008, vol. 77, no. 263, pp. 1599-1613.

DOI: https://doi.org/10.1090/S0025-5718-08-02085-1

[3] Brauchart J. S., Hardin D. P., Saff E. B. The Riesz energy of the Nth roots of unity: an asymptotic expansion for large $N$. Bulletin of the London Mathematical Society, 2009, vol. 41, part 4, pp. 621-633.

DOI: https://doi.org/10.1112/blms/bdp034

[4] Brauchart J. S., Hardin D. P., Saff E. B. The next-order term for optimal Riesz and logarithmic energy asymptotics on the sphere. Contemp. Math, 2012, vol. 578 , pp. $31-61$.

DOI: http://dx.doi.org/10.1090/conm/578

[5] Criado del Rey J. G. On the separation distance of minimal Green energy points on compact Riemannian manifolds. 2019, arXiv:1901.00779.

[6] Dubinin V. N. Condenser capacities and symmetrization in geometric function theory. Basel: Birkhauser/ Springer, 2014, xii +344 pp.

DOI: https://doi.org/10.1007/978-3-0348-0843-9

[7] Dubinin V. N., Kovalev L. V. The reduced modulus of the complex sphere. J. Math. Sci. (New York), 2001, vol. 105, no. 4, pp. 2165-2179.

DOI: https://doi.org/10.1023/A:1011377024516

[8] Dubinin V. N. On the product of inner radii of "partially disjoint" domains. In: Questions of the Metric Theory of Mappings and Its Application, Naukova Dumka, Kiev, 1978, pp. 24-31 (in Russian).

[9] Erdélyi T., Hardin D. P., Saff E. B. Inverse Bernstein inequalities and min-max-min problems on the unit circle. Mathematika, 2015, vol. 61 (03), pp. $581-590$.

DOI: https://doi.org/10.1112/S0025579314000138

[10] Hardin D. P., Kendall A. P., Saff E. B. Polarization optimality of equally spaced points on the circle for discrete potentials. Discrete \& Computational Geometry, 2013, vol. 50 (1), pp. 236-243.

[11] Kuz'mina G. V. Methods of geometric function theory, I, II. St. Petersbg. Math. J., 1997, vol. 9, no. 3, pp. 455-507; no. 5, pp. 889-930.

[12] López-García A., Wagner D. A. Asymptotics of the Energy of Sections of Greedy Energy Sequences on the Unit Circle, and Some Conjectures for General Sequences. Comput. Methods Funct. Theory, 2015, vol. 15, no. 4, pp. $721-750$.

DOI: https://doi.org/10.1007/s40315-015-0140-0

[13] Lebedev N. A. The area principle in the theory of univalent functions. Nauka, Moscow, 1975 (in Russian). 
[14] Marcus M. Radial averaging of domains, estimates for Dirichlet integrals and applications. J. Anal. Math., 1974, vol. 27, pp. 47-78.

Received July 18, 2019.

In revised form, October 14, 2019.

Accepted October 30, 2019.

Published online November 7, 2019.

Far Eastern Federal University

8 Sukhanov str., Vladivostok, 690090, Russia

Institute of Applied Mathematics, Far Eastern Branch of RAS

7 Radio str., Vladivostok, 690041, Russia

E-mail: dubinin@iam.dvo.ru 